\title{
Integrated Machine Health Monitoring Application: A Cloud-Based Approach
}

\author{
Rajeswari N, AnanyaMrituanjaya A \\ \{*nrj.mca@psgtech.ac.in, A_Ananya_M@cat.com\}
}

Assistant Professor (Sr.Gr), Department of Computer Applications, PSG College of Technology, Coimbatore -641 004,Tamilnadu, India ${ }^{1}$ PG Student, Department of Computer Applications, PSG College of Technology, Coimbatore -641 004,Tamilnadu, India ${ }^{2}$

\begin{abstract}
Health Equipment Insights (HEI) is a cloud hosted, subscription-based web application. In this application, the health of the various machines such as trucks and loaders are monitored through various parameters and useful insights are given to the customers and dealers who own the equipments. There are three kinds of users - dealers, customers and CAT users. In order to access the HEI application, the customers and dealers must have subscribed to the service. The insights given to the users are productivity information like engine high speed shutdown, and high gas temperature, along with other information. It also provides utilization details such as the total amount of fuel consumed, the number of hours and performance indicators such as downtime. The users can also schedule to receive the information in form of reports on daily, weekly or monthly basis. These insights help in increasing the productivity at the mining sites and for utilizing the costs efficiently. They also help in providing health insights of various components inside the equipment. This information can be used to analyze the components inside the equipment thereby reducing the wear and tear. The preliminary driving factor as far as this application is concerned, is the urge to deliver more value to the customer by providing more user-friendly, easy to use interface with richer user interface experience along with additional features such as shared dashboard, feedback maintenance.
\end{abstract}

Keywords - Content Delivery System, Mining, Data Logs, Widgets

\section{Introduction}

Machine's performance and health is monitored with the help of binary data generated with the electronic sensors and control system available in the equipment. Health Equipment Insights is a cloudhosted, internet-accessible data visualization and reporting tool with customizable, interactive data dashboards and intuitive drill-down functions. This binary data is stored locally in the customer's environment. The data from the customer's environment is fetched as files and stored in the Database. The Health Equipment Insights application retrieves the data by hitting the API. This data is used by the application to enable the customer to analyze the equipment's performance and productivity[1]. This application helps to improve the performance of the assets by showing its productivity in various environments, including mining, construction equipments as shown in below fig 1 and fig 2. 


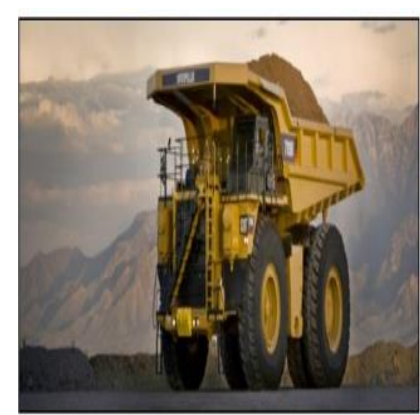

Fig 1.CatConstructionEquipment

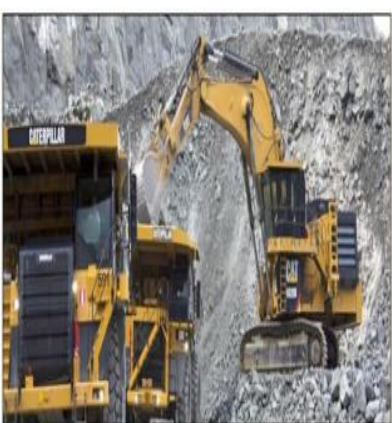

Fig 2.Machinefor Gradingand Loading

\section{Proposed system}

The objective of this application is to fetch equipment data from a central database of the organization. Content Delivery System (CDS) receives data files from the electrical equipment attached to the customer's machine. These files are then processed and stored in central repository. This data from the database would be used by the application to visualize the equipment's health and performance. This enables the customers to monitor the equipment's health[2], performance and productivity and allows them to make informed management decisions. This is achieved by updating the plug-in and improving the authentication in the client side and in the backend the technologies used are Java 8, Spring Boot and Cloud Storage.

The application can upload files, search for existing files in the server, updating privacy settings, adding comments on the files. Comparison of two parse able reports is possible but often depending on multiple often conflicting factors. The application is a web solution which allows users to track their assets productivity and view productivity data through widget feature. Productivity of a machine depends on its health and performance.

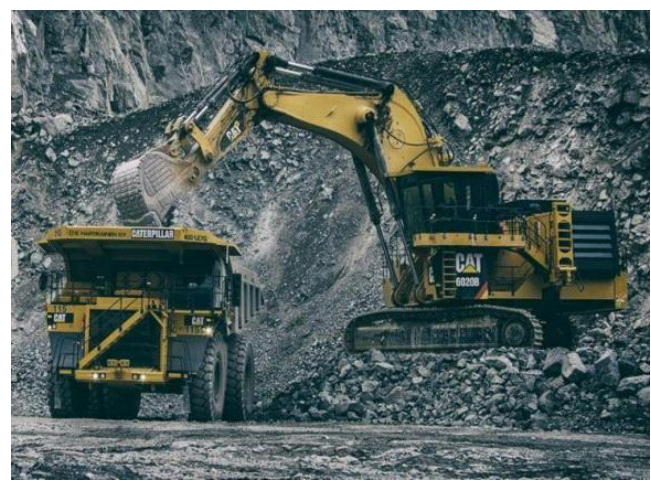

Fig 2.CAT Machines in Mining Process

In the mining environment, for a process, there could either be one equipment involved or a number of equipments involved, as shown in Fig 2. Hence, the process differs in accordance to the scenarios. Health Equipment Insights application retrieves the machine's data by hitting the API provided by the CDS. This data is used by the application to enable the customer to analyze the equipment's performance and productivity. This application helps to improve the performance of the assets by showing its productivity in various environments.

- Application Time - A breakdown of the amount of time the loader is spending in each application or segment. 
- Application Fuel - A breakdown of the fuel the loader is burning in each application or segment. Data options include total fuel burned and fuel burned per hour.

- Application Payload - A breakdown of the payload moved in each application of segment. Data options include Productivity (payload/hr), Efficiency (payload/fuel), and payload distribution.

- Application Cycle- A breakdown of cycle information in each application or segment. Data types include cycle count, fuel burned per cycle, and average cycle times.

- Application Segment - A breakdown of average segment time within selected applications.

- Application Totals - A table showing total cycles, time, fuel, distance and payload information for all applications.

- Application Averages - A table showing average cycles, time, fuel, distance and payload information for all applications.

- Day Tracker - A chronological graph of applications throughout a given day. Day tracker only displays one days' worth of information even when multiple days are selected.

- Application Payload Summary - A table showing a breakdown of payload data by material ID and truck ID.

- Application Map - An interactive map view showing the location of each application during the selected time period.

The Health Application Insights tool is made up of widgets to display time and fuel information by application as well as transactional payload information. When the tool is opened a default layout of widgets is displayed. Widgets can be dragged and dropped into the Health Equipment Insights dashboard Dashboards of widgets can be saved and restored in future using the toolbar at the top. Some widgets have settings to change data and plot settings using the gear icon.

The goals are to improve the legacy system, since it did not provide the users with richer business intelligence. User Interface of the system can be improved and modified to the latest technologies. The performance of the existing system can be improved to make it faster, better and more efficient to be able to better utilize very limited resources such as memory, caches, networks.

- Improved performance

- $\quad 80 \%$ improvement in asset on-boarding time

- $\quad 75 \%$ improvement in DSP Work list load time

- Gained capacity for ongoing growth of connected assets.

\section{SYSTEM DESIGN}

The front-end application follows the MVC Architecture to create complex web applications where front-end is capable of utilizing multiple back-end REST APIs. This allows the front-end to be created in a view specific model as shown in Fig.3.

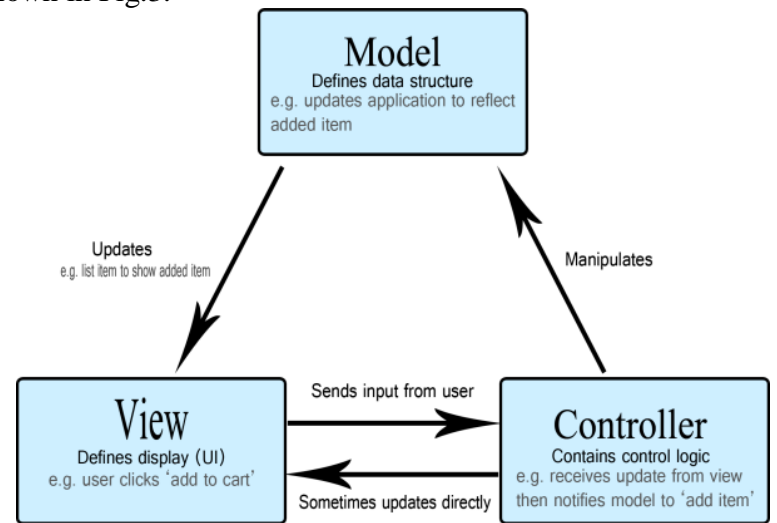

Fig 3.MVC Architecture 
The views handles how application looks to the user. It is mainly designed using HTML templates, SCSS or CSS styling and Angular[3]-[6] directives. The controllers responsible for attaching the data that is generally retrieved from back-end or can be an asset of the app to the views.

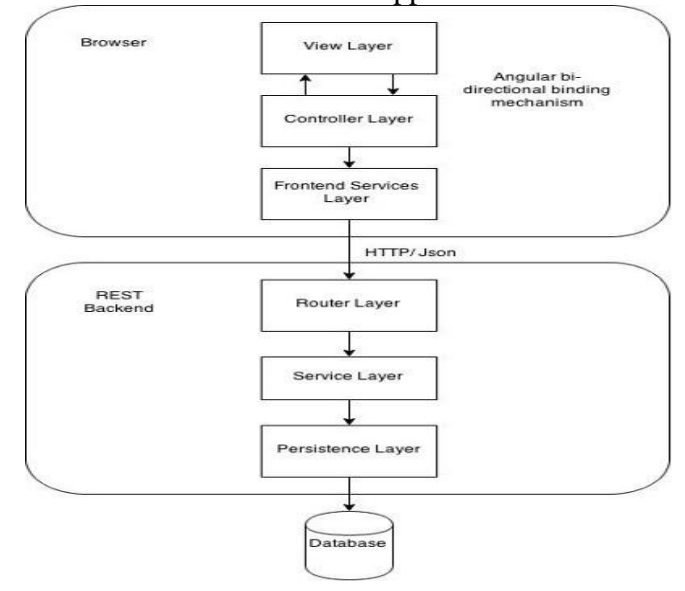

Fig 4. Angular working with Back-end REST API

They are also responsible for reactions and responses of both view and model when either one of them changes. A group of services in the app that allows the back-end to communicate with the application and can influence the controllers by injecting into it when needed. The back-end is designed and developed using Hibernate, Spring boot, Java 8. This allows the back-end services to be divided into micro-services. The interaction between front end and back end is depicted in Fig 4

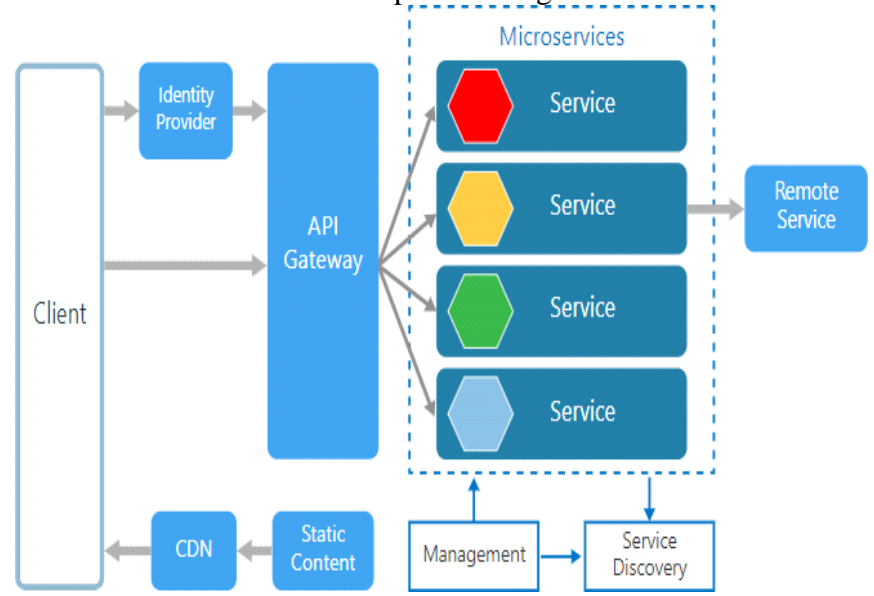

Fig 5.Microservices Architecture in Application

The application functions in micro services architecture design. The micro services architecture in the application is shown is Fig 5. This allows the application to be created in such a way where the front-end and back-end be independently designed and developed. Communication way such as request-response formats, headers, types of headers to be allowed, control of content-type, authentication processes has to be decided and negotiated between the two parties. It also helps with discovering where exactly the error is coming from and resolving it. This also allows creating a system where if one system goes down it doesn't lead to the downfall of the entire application.

\section{Overall Architecture}


The overall architecture shows the various back end and front end components glued to each other for efficient functioning of the system. The users or subscribers enter into the application by accessing the website URL. They, at first interact with the Angular[3]-[6] components. The changes made by the users are handled by the Node JS components. The requests are then hit though the API calls made to the backend. The backend consists of various micro services in form of APIs. The APIs that were created for various purposes then adhere to the requests and hit the CDS for the data. The data that has been collected from CDS is analyzed and converted into meaningful data and then the data is sent back to the front end through the Node JS components, as shown in Fig 6. The Node JS and Angular[3]-[6] then convert it into data visualizations, as required for the user.

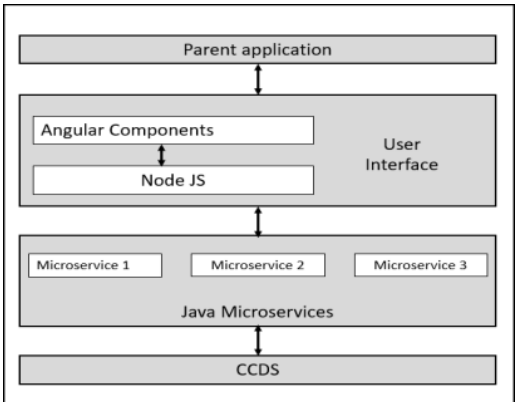

Use Case Diagram

Fig 6. Overall Architecture of the Application

The user case diagram for the user depicts the control the user has over the system. The user can login, view, manipulate, set preferences and give feedback, as shown in Fig 7.

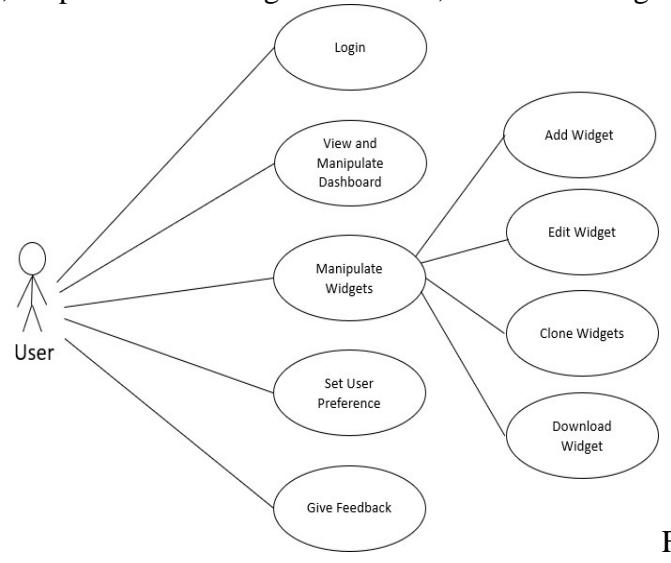

Fig 7. Use Case Diagram

\section{Sequence Diagram}

The sequence diagram elaborates another view of the work flow of the system. It shows the various entities involved in the system. They include customer, user interface layer, microservice layer and the CDS as shown in Fig.8. 


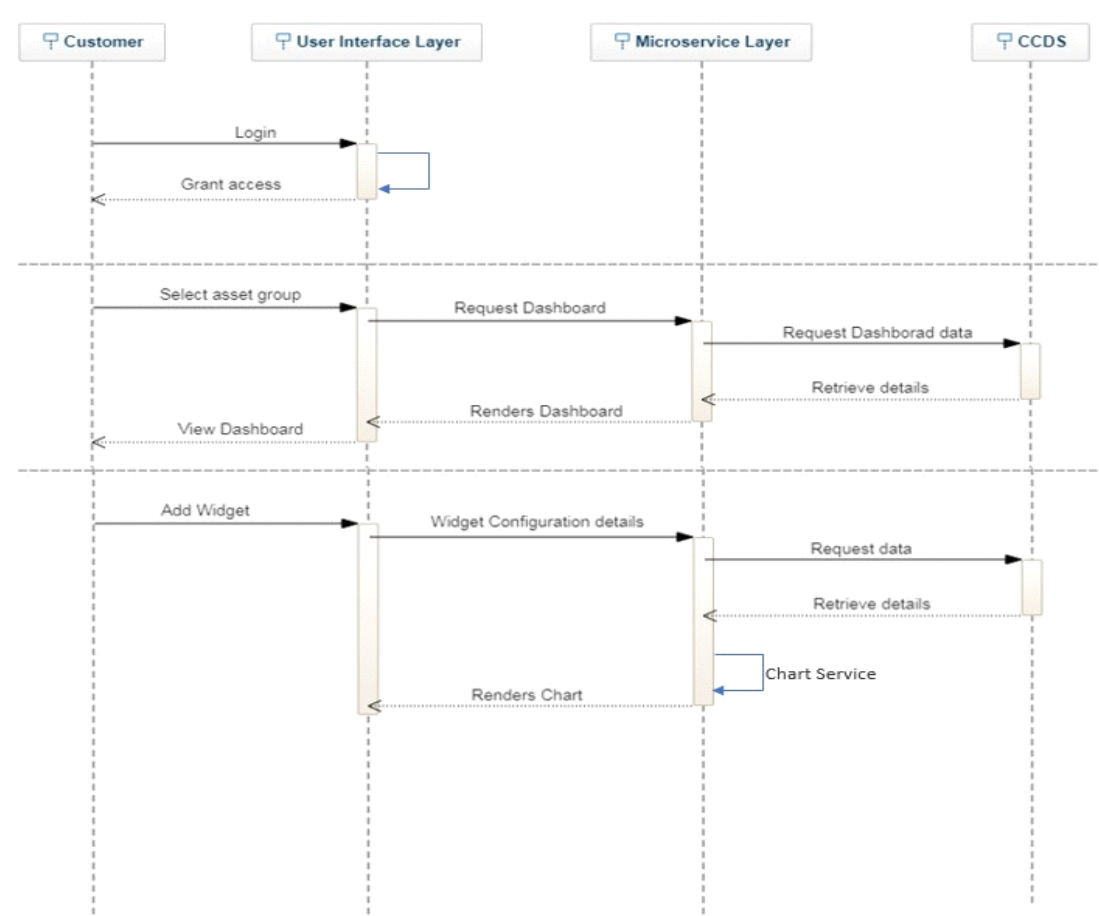

Fig 8. Sequence Diagram

The data exchange between the application and the user is shown in Fig.9. It also shows the data validation points of the application. This allows the enhancement of the security features there by increasing the security threshold of the system.

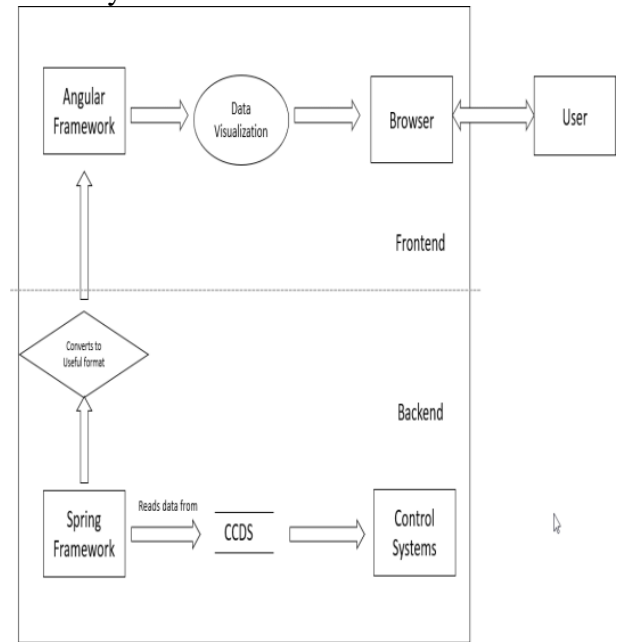

Fig. 9 Exchange of data between App and User 


\section{SYSTEM WORKFLOW AND COMPONENTS}

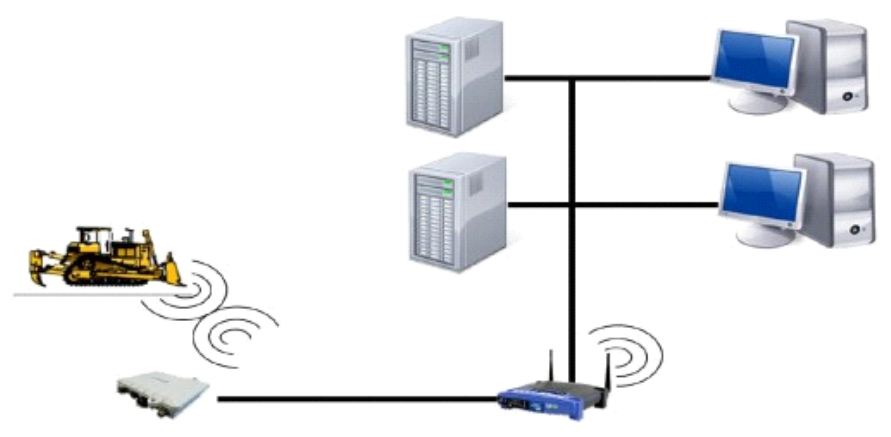

Fig.10 System Components

Health Equipment Insights can only be accessed by a user with CWS account. The various components are shown in Fig 10. The application widgets are as follows

\section{Home Page}

The Health Equipment Insights home page display the following:

- Dashboard - The Dashboard tab allows the user to view three different types of dashboards- a Summary Dashboard, a Map Dashboard and an Individual Machine View Dashboard.

- Asset Target - The Asset Target tab allows the user to view the assigned equipment along with the target list by selecting the dealer or group. It allows the user to find the data easily so that the user can group it accordingly and apply the same to create widgets.

- $\quad$ Manage Files - The Manage Files tab is used to view any manually uploaded files.

- Scheduled Reports - The Scheduled Reports tab is used to view, edit and delete the available scheduled reports.

- Datalogger - The Datalogger tab includes two options: standard datalogger and continuous datalogger. The standard datalogger provides a summary of channel details when the datalogger was turned on. The continuous datalogger provides a summary of channel details on a 60 minute interval. This feature is included on newer machines.

- Equipment Group - The Equipment Group feature allows the user to create a new group or edit an existing equipment group.

- Date Range Selector - The Date Range Selector is to select the date range of the data used in the widgets.

- Schedule Report - The Schedule Report feature is used to schedule a recurring email distribution of a specific report to a specified list of recipients.

- Add Widget - The Add Widget feature is used to add in widgets to create a customized dashboard.

- $\quad$ My Views - The My Views feature allows the user to view a list of saved dashboards.

- Save View - The Save View feature allows you to save the desired widgets as a dashboard and load the dashboard at any moment for quick reference.

- Book Icon - The User Manual includes in-depth information on Health Management Software.

- Profile Icon - The Profile Icon includes Preferences, Feedback and Log Out. Preferences allows the user to define specific settings for the application. Feedback allows the user to provide feedback on any bugs, glitches, data inconsistencies, lack of functionality or other problems in the application. Log out allows the user to exit from the application.

- Search Icon - The Search Equipment icon allows users to search for the equipment using Serial Number.

\section{Dashboard}


Users can create customized dashboards that allows the user to visualize data in a variety of widgets The Dashboard tab on the Health Equipment Insights home page has the following options:

- Summary View - Includes the user specified default dashboard from preferences with a specified Equipment Group.

- Map View - Includes the specified Equipment Group`s cycle and segment data in chart, detail and map views. Map View gives an overview of Cycle Time, Payload and Fuel Usage while providing equipment location and the option to download cycle details.

- Individual Machine View - Allows the user to view all the available configured asset details from both the dealer and customer apart from any other machines. The asset details include Service Meter, Last Reported Time, Location and File Download Summary.

Equipment Group

An equipment group is a specific set of equipment from either a dealership or customer that can be viewed and analyzed together. Equipment can be used in more than one group and all groups can be access from the summary view dashboard. The first time the user logs into Health Management Software, the user will be prompted to create an equipment group, instructions can be found in the Accessing Health Equipment Insights for the First-Time section.

Date Range Selector

The Date Range Selector is used to select the date range for which the data is to be displayed in the widgets.

Widget

To add a widget:

- $\quad$ From the Health Equipment Insights homepage, click the Add Widget icon.

- $\quad$ Click the category of the desired widget.

- $\quad$ Click the desired widget and click Next.

- Three wizards are used to create widgets in Events: Select Widget, Configure Widget and Confirm and Apply. Complete the widget configuration steps by selecting the arrow icon of each item and selecting the desired option. Once the user had completed each step, click Next.

- Confirm and apply the widget by reviewing the configuration and selecting Add.

My Views

My Views feature allows the user to view a list of saved dashboards under the dashboards tab. The My Views feature also allows the user to share saved dashboard with other users under the shared dashboards tab. Users can edit, delete and share the saved dashboards, and save and reject the shared dashboards, and also search the saved and shared dashboards.

Asset Target

The Asset Target allows user to view the assigned equipment along with target list by selecting the dealer or group. It allows user to find the data easily so that the user can group it accordingly and apply the same to create widgets.

\section{Schedule Report}

The Schedule Report runs on a scheduled interval to trigger the report of the currently-viewed dashboard to the provided recipients. The scheduled report is sent as an email to the provided recipients. The Scheduled Reports option allows users to create, edit and delete the available scheduled reports.

\section{Datalogger}

The Datalogger option allows the user to view the summary of Channel details with Unit, Channel Group, Minimum and Maximum values for the configured equipment group. The Datalogger tab in the Health Equipment Insights home page has the following options:

Standard Datalogger - Includes channel details when the user or operator turns on the datalogger. The standard datalogger widget provides summary of channel details with unit, channel group, minimum and maximum values for the configured equipment group in a table.

Continuous Datalogger - Includes channel details on 60-minute intervals. This feature is included on newer machine models. The continuous datalogger widget provides summary of channel details with unit, channel group, minimum and maximum values for the configured equipment group in a table.

Channel Configuration - Allows the user to create a Channel Group for Standard Datalogger, Continuous Datalogger and Trends. The Channel Group provides channels with minimum and maximum 
limit values for the selected assets. The Channel Group can be modified or deleted in the configuration page.

Widgets

The data dashboards are powered by widgets and are therefore highly interactive to allow for a customized user experience. There are various widgets the user can chose from including widgets in the following categories.

Events

Events provides the summary count and description details of event for the selected equipment with event level.

Utilization

The Utilization provides information on the Fuel consumption and Hours during Idle and working time of the configured equipment group.

\section{Time Series}

The Time Series provides summary of Data Logger, Value Point Indicator, Trend and Snapshot for the configured equipment group.

Cumulative

The Cumulative widget provides a summary of Channel count for the configured equipment group and channel.

\section{Histogram}

The Histogram displays the channel with condition, average, and standard deviation for frequency distribution of a continuous set of data.

Productivity

The Productivity widgets helps the users to track their assets productivity and view productivity data in Charts and Table.

Inspections

The Inspections widget allows the user to view the list of checklists with its status for the configured equipment group.

Fluids

Fluid analysis reports provided by the Fluids Lab for Oil, Coolant, and Fuel samples are available in the Fluids widget. The data is displayed in both Chart and Detail views.

Map

Map widget allows the user to view the last reported location and Event Map for the configured Equipment Group.

\section{Manage Files}

The Manage Files allows user to view any uploaded files and upload new files.

Preferences

The preferences option allows the user to define specific settings for the application. The following settings can be configured: Dashboard, Location/Time and Unit of Measure.

\section{CONCLUSION}

Health Equipment Insights is a cloud-hosted, internet-accessible visualization and reporting application that goes well beyond providing the data user needs. Customizable, interactive data dashboards and intuitive drill-down functions allow users to quickly and easily navigate large volumes of machine data to gain the equipment insights to diagnose an event and make informed maintenance decisions.

Decisions that help increase safety and maximize asset reliability by improving Mean Time Between Stoppages (MTBS) and reducing Mean Time to Repair (MTTR). Health Equipment Insights visualizes and reports machine health data, allowing maintenance staff to diagnose equipment events, monitor critical machine parameters and analyze operational trends and patterns.

This Mining Software also provides access to a variety of application and production data to assist operational personnel with tracking production related Key Performance Indicators (KPIs). In addition, by 
combining IMS and Product Link data with inspection and fluids data, Health Equipment Insights gives you access to a variety of data sets that help you track production, fuel burn or overall machine performance.

\section{References}

[1]. Nadakatti Mahantesh,Parida Aditya,Uday Kumar ," Integrated machine health monitoring: a knowledge based approach", International Journal of Systems Assurance Engineering and Management, Volume 5,Issue 3,2013.

[2]. Candell O, Karim R, Soderholm P ,'eMaintenance-Information Logistics for Maintenance Support", Robotics and Computer Integrated Manufacturinig, Volume 25,Issue 6, pp.937-944, 2009.

[3]. Wutthichai Chansuwath, Twittie Senivongse, "A Model-Driven Development of Web Applications Using Angular JS Framework", IEEE/ACIS 15th International Conference on Computer and Information Science (ICIS), 2016.

[4]. Abdur Rahman, S. Chitra Devi, "A Framework For Ultra-Responsive Light Weight Web Application Using AngularJS”, Online International Conference on Green Engineering and Technologies (ICGET), 2015.

[5]. Nina Fat, Marijana Vujovic, Istvan Papp, Sebastian Novak, "Comparison of AngularJS Framework Testing Tools", Zooming Innovation in Consumer Electronics International Conference (ZINC), 2016

[6]. Sanja Delcev, Drazen Draskovic, "Modern JavaScript frameworks: A Survey Study", Zooming Innovation in Consumer Technologies Conference (ZINC), 2018. 